Revue de droit comparé du travail et de la sécurité sociale

$3 \mid 2020$

La Directive 2019/1158 du 20 juin 2019 concernant l'équilibre entre vie personnelle et vie privée des parents et des aidants

\title{
Mesures sociales prises pour lutter contre la propagation du Covid 19
}

\author{
Yaroslava Genova
}

\section{OpenEdition}

Journals

Édition électronique

URL : https://journals.openedition.org/rdctss/1035

DOI : $10.4000 /$ rdctss. 1035

ISSN : 2262-9815

Éditeur

Centre de droit comparé du travail et de la sécurité sociale

Édition imprimée

Date de publication : 1 novembre 2020

Pagination : 196-199

ISSN : 2117-4350

Référence électronique

Yaroslava Genova, «Mesures sociales prises pour lutter contre la propagation du Covid 19 », Revue de droit comparé du travail et de la sécurité sociale [En ligne], 3 | 2020, mis en ligne le 01 novembre 2021 consulté le 11 novembre 2021. URL : http://journals.openedition.org/rdctss/1035 ; DOI : https:// doi.org/10.4000/rdctss. 1035

\section{(c) (i) $\odot$}

Revue de droit comparé du travail et de la sécurité sociale est mise à disposition selon les termes de la Licence Creative Commons Attribution - Pas d'Utilisation Commerciale - Pas de Modification 4.0 International. 


\section{YAROSLAVA GENOVA}

Faculté de Droit de L'Université de Plovdiv «PAissil Hilendarski »

\section{MESURES SOCIALES PRISES POUR LUTTER CONTRE LA PROPAGATION DU COVID 19}

Afin de prévenir une crise médicale liée à la propagation du nouveau virus COVID-19, le Parlement bulgare a pris la décision de mettre le pays provisoirement en état d'urgence (procédure prévue par la Constitution) dès le 13 mars 2020, peu après le tout premier cas enregistré d'une personne infectée.

En complément, une loi spéciale («Loi sur les mesures et les actions durant l'état d'urgence, et sur les moyens de surmonter ses effets ") ${ }^{1}$ a introduit diverses mesures dans plusieurs domaines sensiblement liés aux répercussions de la lutte contre l'épidémie (dont l'arme essentielle fût l'isolation sociale), notamment dans les domaines du droit du travail et du droit de la sécurité sociale.

Cette loi fût adoptée comme législation temporaire. Le 13 mai dernier en effet, l'état d'urgence a pris fin et le Parlement ne l'a pas prolongé. Les mesures d'isolation préventive furent sensiblement relâchées, mais la maladie ellemême s'était déjà révélée comme une pandémie aux conséquences inconnues. Dès lors, le Ministre de la santé a déclaré le territoire national en « situation épidémique exceptionnelle ", ce qui a permis de prolonger l'application des mesures sanitaires inhérentes à la lutte complexe contre COVID-19.

Parallèlement, la loi temporaire a été convertie en loi permanente de manière à faire appliquer, autant que nécessaire, les autres mesures et actions liées aux perturbations de la vie sociale et économique dues à la pandémie.

L'article 7 de la loi sur les mesures et les actions durant l'état d'urgence et sur leurs conséquences, a ostensiblement modifié l'une des règles essentielles relative à la portée de l'accord des deux parties à un contrat de travail.

En effet, face à cet impératif de santé publique, l'employeur a obtenu le droit de modifier unilatéralement la clause déterminante du lieu du travail et d'imposer au travailleur, qu'il soit salarié ou cadre, d'exécuter ses obligations de travail à domicile ou en télétravail. Pour ce faire, la seule condition résidait dans le fait qu'il existe une possibilité réelle d'exécuter son activité en dehors du lieu habituel de travail.

1 Journal officiel $n^{\circ} 28$ du 24 mars 2020, en vigueur au 13 mars 2020; modification des $n^{\circ} 34$, $38,44,55$ et 60 . 
Cette règle a été précisée via l'introduction d'un nouvel article $120 \mathrm{~b}$ dans le Code du travail. Bien que permanente, cette norme réserve l'introduction du travail à domicile - ou du télétravail - à un ordre donné par l'employeur en cas d'état national d'urgence, ordonné par le Parlement. Des précisions ont également été apportées concernant la rémunération et les termes essentiels du contrat du travail, ces deux domaines ne pouvant être modifiés au motif du travail à domicile ou du télétravail.

En outre, l'employeur se voit obligé de fixer par écrit les conditions liées aux spécificités du travail à domicile ou du télétravail, notamment la couverture des frais tels que le loyer, le coût de l'électricité, la connexion internet, etc.

L'article 120c du Code du travail prévoit un droit de fermeture temporaire des lieux de travail sur ordre de l'employeur en raison d'un état d'urgence décrété par le Parlement.

L'article 267a complète cette règle en précisant que ces périodes d'interruption du travail sont rémunérées comme des jours ouvrés.

En outre, I'article 138a, alinéa 2, autorise l'employeur -toujours dans les conditions exceptionnelles - à introduire unilatéralement le travail à temps partiel.

La dernière disposition de cette nouvelle « législation du travail en cas de force majeure » concerne les congés annuels payés. L'article 173a du Code du travail donne droit à l'employeur qui a fermé son site de travail en raison d'un état d'urgence établi par le Parlement, à contraindre ses salariés à prendre leur congé annuel durant cette période.

Parallèlement, si l'activité se poursuit en dépit d'un état d'urgence, l'employeur se voit lui-même obligé d'octroyer sans conditions le congé au travailleur qui en fait la demande, dès lors que ce dernier fait partie d'un groupe social particulièrement atteint par la situation du COVID-19 :

- les femmes enceintes;

-les jeunes de 16 à 18 ans ;

- les personnes handicapées ou atteintes de maladies chroniques graves ;

- les parents d'enfants âgés de moins de 12 ans ou souffrant d'un handicap.

Ces différentes règles ont donné des effets positifs ostensibles entre mimars et mi-mai 2020, i.e. durant «l'état d'urgence »- quand l'objectif national était de réduire au maximum le nombre de personnes atteintes du COVID-19 - au prix de mesures particulièrement lourdes en termes d'isolation sociale (fermeture des écoles, des grandes entreprises, de la plupart des commerces et services, des lieux culturels et de plaisance, etc.).

S'agissant des professeurs des écoles, la Loi du 13 mars 2020 a expressément prévu la mise en place du télétravail pour les enseignants - de droit et sans obligation de consentement - jusqu'à la fin de l'année scolaire 2019-2020. 
Corrélativement, le Ministre de l'éducation dispose du pouvoir de modifier provisoirement, si besoin en était, le lieu et les conditions de travail du corps des enseignants.

Nonobstant une rentrée scolaire 2020-2021 relativement normale, des discussions ministérielles sont en cours sur la possibilité de poursuivre un enseignement à distance en faveur de certaines catégories d'élèves particulièrement vulnérables.

Par deux ordonnances successives du Gouvernement ${ }^{2}$, des mesures d'aide financière ont été mises en place pour les entreprises ayant permis la sauvegarde des postes de travail en dépit des pertes économiques liées à la " crise COVID-19 " (que ce soit consécutif à la fermeture temporaire imposée des lieux de travail ou à la diminution des gains).

Cette aide est plus populairement connue sous le nom de «60:40»: en effet, les employeurs satisfaisant les conditions du programme d'aide administré par l'Agence de l'emploi peuvent recevoir de l'Etat une somme équivalente à 60\% du salaire de chacun des travailleurs dont l'emploi a échappé à un licenciement économique, ainsi que $60 \%$ du taux des cotisations sociales obligatoires.

De ce fait, le coût de la main-d'œuvre restant à la charge des employeurs bénéficiaires n'est plus que de $40 \%$ du coût total.

Le programme « 60:40 » est supposé fonctionner jusqu'au 30 septembre 2020. Toutefois, les conditions requises pour en bénéficier se sont avérées inadaptées aux secteurs du transport et du tourisme, pourtant très lourdement impactés par la situation pandémique.

Aussi, un programme spécial dénommé « Développement des ressources humaines " a été mis en œuvre et doit permettre, à court terme, d'aider à la sauvegarde de 22000 postes de travail dans ces deux secteurs.

Comme ses conditions et exigences se sont avérées inadéquates pour les secteurs des transports et du tourisme, particulièrement affectés par la situation pandémique, un programme spécial à court terme est en cours, financé dans le cadre du programme opératif « Développement des ressources humaines ", visant à sauvegarder 22000 postes de travail dans ces secteurs.

Chaque employeur ou travailleur prouvant une diminution de gains d'au moins $20 \%$ - incontestablement engendrée par la crise actuelle - recevra donc directement 290 leva $^{3}$ en complément de son salaire, dès lors que l'emploi concerné a effectivement été sauvegardé, et que le travailleur s'engage à assurer son travail durant une période future au moins égale à la moitié de la période pour laquelle l'aide sera octroyée.

$2 \quad N^{\circ} 55$ du 30 mars 2020 et n¹51 du 3 juillet 2020.

3 Environ 145 euros. 


\section{BULGARIE}

Les règles et les mesures ici décrites ne relèvent, hélas, que de "la première vague » législative imposée par l'urgence suite à la pandémie de COVID-19.

Actuellement, d'autres amendements et de nouvelles dispositions en matière de législation sociale sont en cours l'élaboration et s'orientent davantage sur la suite de cette situation pandémique persistante, notamment sur le marché du travail. 